УДК 537.21, 539.217, 620.3

\title{
Simple Mathematical Model of Conducting Nanopore
}

\author{
Oleg A. Zolotov* \\ Viktor E. Zalizniak ${ }^{\dagger}$ \\ Institute of Mathematics and Computer Science \\ Siberian Federal University \\ Svobodny, 79, Krasnoyarsk, 660041 \\ Russia
}

Received 13.11.2017, received in revised form 28.03.2018, accepted 20.06.2018

A simple model of conducting nanopore is presented in the paper. The model does not require solution of the Poisson equation for the potential. This model is intended for use in simulation of transport phenomena of charged particles in conducting nanopores by the method of molecular dynamics. The model can be easily applied to an arbitrary shape nanopore.

Keywords: conducting nanopore, ion transport, molecular dynamics.

DOI: 10.17516/1997-1397-2018-11-4-505-512.

\section{Introduction}

Nanopores are pores with a diameter in the range of nanometers. Channels of this size can be now manufactured artificially. There is great scientific interest in nanopores due to their importance and possible applications [1,2]. Artificial nanopores can be used to imitate some of the functions of biological nanopores. They also have interesting applications in the field of nanofluidics [3]. Among these applications are desalination, ion sieves [4], sensors for biological agents and sequencing of DNA or RNA [5].

Various shapes of artificial nanopores have been developed with the use of various manufacturing techniques, such as ion-track-etching, laser, electron beam technologies, anodic oxidation method etc. [6]. There are five types of nanochannels: cylindrical, hourglass, cigar-like, bullet-like and conical [7].

Various types of nanopores and nanochannels have attracted significant interest in recent years. We focus our attention on conducting nanopores. For example, such nanopores can be manufactured by integrating carbon structures into a proper nanoporous material. Multi wall carbon nanotube can be fabricated inside the pore and at the surface of anodic aluminum oxide membrane by chemical vapor deposition method [8]. Aligned metallic single walled carbon nanotubes were grown on a silicon wafer by chemical vapour deposition [9]. Synthesis of conducting carbon tubules inside the pore of anodic aluminum oxide membrane was considered [10]. These structures may be of interest in terms of fabricating electrically-switchable permselective membranes.

The purpose of this paper is to present simple model of a conducting nanopore. For the sake of simplicity cylindrical nanopore is considered. This model is intended for use in fundamen-

\footnotetext{
*ozolot @mail.ru

†vzalizniak@mail.ru

(c) Siberian Federal University. All rights reserved
} 
tal studies of transport phenomena of charged particles (ions and charged protein residues) in conducting nanopores.

In Section 2 of the paper we briefly consider problem formulation in the context of continuum electrostatics. A simple model of conducting nanopore that does not require solution of the Poisson equation for the potential is presented in Section 3. In Section 4 we verify the accuracy of the proposed model by calculating the self-capacitance of conducting cylindrical shell and comparing it with the reference data. Simulation of interaction of singly charged ions with some conducting nanopore is presented in Section 5. In section 6 we summarize our results and make some conclusions.

\section{Problem formulation}

Let us consider a membrane with a nanopore. The membrane is modeled as a uniform dielectric slab of width $L$ located between $z=0$ and $z=L$. A nanopore is a cylindrical hole of radius $a$ and length $L$. It is convenient to set up a cylindrical coordinate system $(z, \rho, \theta)$ with the origin located on the symmetry axis of the nanopore at $z=0$.

Consider the ion transport problem in the context of continuum electrostatic model. Suppose that an ion is located at an arbitrary position $\mathbf{r}^{*}$. The electrostatic potential $\varphi(z, \rho, \theta)$ inside and outside the nanopore satisfies the Poisson equation

$$
\begin{gathered}
\Delta \varphi=-\frac{1}{4 \pi \varepsilon \varepsilon_{0}} \sum_{n=1}^{N} q_{n} \delta\left(\mathbf{r}-\mathbf{r}_{n}^{*}\right), \\
\mathbf{r}=(z, \rho, \theta),
\end{gathered}
$$

where $\Delta$ is the Laplace operator, $q_{n}$ is the ion charge, $N$ is the number of ions, $\delta($.$) is the Dirac$ delta function. Equation (1) should be solved over all space except the domain $\{0 \leqslant z \leqslant L, \rho>a\}$ with the following boundary conditions:

1) $\varphi(z, a, \theta)=u$,

2) $\varphi(z, \rho, \theta) \rightarrow 0$ at $\rho \rightarrow \infty, z \rightarrow \pm \infty$,

3) normal to the surface of dielectric components of electric displacement field are continuous, where $u$ is the specified voltage. Considering the transport phenomena of charged particles, we are interested in the force acting on an ion from the conducting nanopore that is expressed as $\mathbf{f}=-q \nabla\left(\varphi\left(\mathbf{r}^{*}\right)\right)$.

Solution of equation (1) with the specified boundary conditions presents a difficult problem. The problem becomes even more complicated if we consider other nanopore shapes than cylindrical shape. Next we present a simple and universal model of conducting nanopore that does not require solution of the Poisson equation for the potential.

\section{Mathematical model of conducting nanopore}

Let us approximate the electric field from the nanopore surface charge with the electric field from fictitious point charges distributed on the surface of the nanopore. The fictitious charges are positioned in specified locations on the nanopore surface. Values of these charges are determined from the potential specified at charge locations. Fictitious point charges $g_{k}, k=1, \ldots, K$, are located at points $\mathbf{r}_{k}$. Then potential at the point $\mathbf{r}_{n}, n=1, \ldots, K$ is 


$$
\varphi\left(\mathbf{r}_{n}\right)=u=\frac{1}{4 \pi \varepsilon \varepsilon_{0}} \sum_{k \neq n}^{K} \frac{g_{k}}{\left|\mathbf{r}_{n}-\mathbf{r}_{k}\right|}+\frac{1}{4 \pi \varepsilon \varepsilon_{0}} \sum_{m=1}^{M} \frac{q_{m}}{\left|\mathbf{r}_{n}-\mathbf{r}_{\mathrm{c}, m}\right|},
$$

where $q_{m}$ is the charge of external charged particle, and $\mathbf{r}_{c, m}$ is the position of external charged particle, $m=1, \ldots, M$. Vector of fictitious point charges $\boldsymbol{g}=\left(g_{1}, \ldots, g_{K}\right)$ satisfies the system of linear equations

$$
A g=b,
$$

where

$$
\begin{gathered}
\boldsymbol{A}=\left\{a_{n k}\right\}, \quad a_{n k}=\frac{1}{4 \pi \varepsilon \varepsilon_{0}}\left\{\begin{array}{l}
\frac{1}{\left|\mathbf{r}_{n}-\mathbf{r}_{k}\right|}, \quad n \neq k \\
0 \quad, \quad n=k
\end{array},\right. \\
\boldsymbol{b}=\left\{b_{n}\right\}, \quad b_{n}=u-\frac{1}{4 \pi \varepsilon \varepsilon_{0}} \sum_{m=1}^{M} \frac{q_{m}}{\left|\mathbf{r}_{n}-\mathbf{r}_{c, m}\right|} .
\end{gathered}
$$

Matrix $\boldsymbol{A}$ is nonsingular matrix (see Appendix for proof).

The electric field of a charged particle approaching a conducting surface causes redistribution of surface electrons in a region closest to the charged particle, in order to screen the field induced by the charged particle. When the distance between charged particle and the nanopore surface is comparable with the average distance between fictitious point charges $g_{k}$ one can introduce additional fictitious point charges $\boldsymbol{p}=\left(p_{1}, \ldots, p_{J}\right)$. They are located on the nanopore surface at points $\mathbf{r}_{p, l}, l=1, \ldots, J$, where $J$ is the number of charged particles that are close to the nanopore surface. Points $\mathbf{r}_{p, l}$ are determined from the normal projection of coordinates of corresponding charged particles $r_{c, m}$ on the nanopore surface. Additional fictitious charge $p_{l}$ is introduced only when

$$
\min _{k}\left|\mathbf{r}_{p, l}-\mathbf{r}_{k}\right|>0.3 \min _{k, n}\left|\mathbf{r}_{n}-\mathbf{r}_{k}\right| .
$$

Then instead of system (2) we have the following system

$$
\begin{aligned}
& \boldsymbol{A}\left(\boldsymbol{g}+\boldsymbol{g}^{*}\right)+\boldsymbol{S} \boldsymbol{p}=\boldsymbol{b}, \\
& \boldsymbol{S}^{T}\left(\boldsymbol{g}+\boldsymbol{g}^{*}\right)=\boldsymbol{d},
\end{aligned}
$$

where $\boldsymbol{g}^{*}$ is the adjustment to main fictitious charges, and matrix $\boldsymbol{S}$ and vector $\boldsymbol{d}$ are

$$
\begin{gathered}
\boldsymbol{S}=\left\{s_{l k}\right\}, \quad s_{l k}=\frac{1}{4 \pi \varepsilon \varepsilon_{0}} \frac{1}{\left|\mathbf{r}_{p, l}-\mathbf{r}_{k}\right|}, \\
\boldsymbol{d}=\left\{d_{l}\right\}, \quad d_{l}=u-\frac{1}{4 \pi \varepsilon \varepsilon_{0}} \sum_{m=1}^{M} \frac{q_{m}}{\left|\mathbf{r}_{p, l}-\mathbf{r}_{\mathrm{c}, m}\right|} .
\end{gathered}
$$

From the first equation of system (3) we obtain

$$
\boldsymbol{g}^{*}=-\boldsymbol{A}^{-1} \boldsymbol{S p}
$$

Upon substituting this relation into the second equation of system (3), we obtain the system of linear equations for additional fictitious charges

$$
\left(\boldsymbol{S}^{T} \boldsymbol{A}^{-1} \boldsymbol{S}\right) \boldsymbol{p}=\boldsymbol{S}^{T} \boldsymbol{g}-\boldsymbol{d} .
$$

Upon solving system (4), we determine main fictitious charges

$$
\boldsymbol{g}+\boldsymbol{g}^{*}=\boldsymbol{A}^{-1}(\boldsymbol{b}-\boldsymbol{S p})
$$




\section{Validation of the model}

To verify the accuracy of the proposed model we calculate the self-capacitance of conducting cylindrical shell of radius aand length $L$, and compare it with the reference data [11]. Then $\boldsymbol{b}=u \boldsymbol{e}, \boldsymbol{e}=(1, \ldots, 1)$ in system $(2)$, and capacitance is

$$
C=\frac{1}{u} \sum_{k=1}^{K} g_{k}=4 \pi \varepsilon \varepsilon_{0} \boldsymbol{e} \boldsymbol{A}^{-1} \boldsymbol{e} .
$$

Let us position fictitious charges at points $\left(z_{n}, a, \theta_{m}\right)$, where

$$
\begin{aligned}
z_{n} & =(n-1) \frac{L}{N-1}, n=1, \ldots, N, \\
\theta_{m} & =(m-1) \frac{2 \pi}{N-1}, m=1, \ldots, N .
\end{aligned}
$$

Then $K=N^{2}$. The results of calculations are presented in Tab. 1 for $5 \leqslant L / a \leqslant 20$ and $N=10, \ldots, 80$. Reference value of the capacitance is denoted as $C_{0}$, and discrepancy between calculated and reference value of capacitance is $d=\left|C-C_{0}\right| / C_{0}$. The results presented in Tab. 1 show that when $L / a>8$ discrepancy is less than $13 \%$ for $N=20$ and discrepancy is less than $5 \%$ for $N=80$. Hence one would expect that when $L / a>8, N \geqslant 30$ parameters of interaction of charged particles with a conducting nanopore are determined with an error less than $10 \%$.

\section{Interaction of ions with a conducting nanopore}

If ion approaches a conducting nanopore its energy of interaction with the nanopore at the point $\mathbf{r}$ is given by

$$
U(\mathbf{r})=\frac{q_{\text {ion }}}{4 \pi \varepsilon_{0} \varepsilon} \sum_{k=1}^{K} \frac{g_{k}}{\left|\mathbf{r}-\mathbf{r}_{k}\right|},
$$

where $q_{\text {ion }}$ is the charge of ion. Electric field produced by the nanopore at the point $\mathbf{r}$ is

$$
\mathbf{E}(\mathbf{r})=\frac{1}{4 \pi \varepsilon_{0} \varepsilon} \sum_{k=1}^{K} g_{k} \frac{\mathbf{r}-\mathbf{r}_{k}}{\left|\mathbf{r}-\mathbf{r}_{k}\right|^{3}} .
$$

Then the force acting on an ion from the conducting nanopore at a point $\mathbf{r}$ is $\mathbf{f}(\mathbf{r})=q_{\text {ion }} \mathbf{E}(\mathbf{r})$.

Let us consider conducting cylindrical nanopore of radius $a=20 \AA$ located between $z=0$ and $z=L=200 \AA$, and apply the proposed model to simulate interaction of ions with the nanopore. Let us calculate energy of interaction (9) and electric field (10) at the location of an ion for positive and negative singly charged ions in vacuum $(\varepsilon=1)$ and in water $(\varepsilon=80)$. We suppose that ions move along the nanopore axis.

Fictitious charges are positioned at points $\left(z_{n}, a, \theta_{m}\right)$ that are given in $(8)$ and $N=50$. The nanopore is set at an electric potential of $1 \mathrm{~V}$.

Results of computations are shown in Figs. 1 and 2.

\section{Conclusions}

A simple model of a conducting nanopore is presented in this paper. The proposed model takes into account polarization of a conducting nanopore due to electric field produced by a 
Table 1. Capacitance of conducting cylindrical shell

\begin{tabular}{|c|c|c|c|c|c|c|c|c|c|}
\hline$N$ & $L / a$ & 5 & 6 & 7 & 8 & 9 & 10 & 11 & 12 \\
\hline \multirow{2}{*}{10} & $C / 2 \pi \varepsilon \varepsilon_{0} a$ & 2.076 & 2.171 & 2.658 & 2.661 & 2.979 & 2.996 & 3.137 & 3.281 \\
\hline & $d, \%$ & 22.6 & 15.7 & 31.0 & 22.1 & 28.2 & 21.6 & 20.6 & 20.0 \\
\hline \multirow{2}{*}{20} & $C / 2 \pi \varepsilon \varepsilon_{0} a$ & 1.830 & 2.104 & 2.079 & 2.419 & 2.605 & 2.752 & 2.891 & 3.029 \\
\hline & $d, \%$ & 8.1 & 12.1 & 2.5 & 11.0 & 12.1 & 11.7 & 11.2 & 10.8 \\
\hline \multirow{2}{*}{30} & $C / 2 \pi \varepsilon \varepsilon_{0} a$ & 1.891 & 1.858 & 2.152 & 2.330 & 2.473 & 2.672 & 2.807 & 2.944 \\
\hline & $d, \%$ & 11.7 & 1.0 & 6.1 & 6.9 & 6.4 & 8.4 & 7.9 & 7.7 \\
\hline \multirow{2}{*}{40} & $C / 2 \pi \varepsilon \varepsilon_{0} a$ & 1.805 & 1.979 & 2.140 & 2.267 & 2.479 & 2.633 & 2.764 & 2.900 \\
\hline & $d, \%$ & 6.7 & 5.5 & 5.5 & 4.1 & 6.7 & 6.8 & 6.3 & 6.1 \\
\hline \multirow{2}{*}{50} & $C / 2 \pi \varepsilon \varepsilon_{0} a$ & 1.737 & 1.996 & 2.128 & 2.465 & 2.426 & 2.614 & 2.737 & 2.874 \\
\hline & $D, \%$ & 2.7 & 6.4 & 4.9 & 13.1 & 4.4 & 6.1 & 5.2 & 5.1 \\
\hline \multirow{2}{*}{60} & $C / 2 \pi \varepsilon \varepsilon_{0} a$ & 1.792 & 1.933 & 2.119 & 2.292 & 2.435 & 2.630 & 2.720 & 2.856 \\
\hline & $D, \%$ & 5.9 & 3.0 & 4.5 & 5.2 & 4.8 & 6.7 & 4.6 & 4.4 \\
\hline \multirow{2}{*}{70} & $C / 2 \pi \varepsilon \varepsilon_{0} a$ & 1.763 & 1.945 & 2.115 & 2.267 & 2.403 & 2.516 & 2.707 & 2.843 \\
\hline & $d, \%$ & 4.2 & 3.6 & 4.2 & 4.1 & 3.4 & 2.1 & 4.1 & 4.0 \\
\hline \multirow{4}{*}{80} & $C / 2 \pi \varepsilon \varepsilon_{0} a$ & 0.793 & 1.467 & 2.119 & 2.252 & 2.412 & 2.540 & 2.697 & 2.833 \\
\hline & $d, \%$ & 53.2 & 21.8 & 4.5 & 3.4 & 3.8 & 3.1 & 3.7 & 3.6 \\
\hline & $C_{0} / 2 \pi \varepsilon_{0} a$ & 1.692 & 1.877 & 2.029 & 2.179 & 2.324 & 2.464 & 2.601 & 2.735 \\
\hline & $L / a$ & 13 & 14 & 15 & 16 & 17 & 18 & 19 & 20 \\
\hline \multirow{2}{*}{10} & $C / 2 \pi \varepsilon \varepsilon_{0} a$ & 3.424 & 3.564 & 3.701 & 3.835 & 3.965 & 4.093 & 4.217 & 4.339 \\
\hline & $d, \%$ & 19.5 & 19.1 & 18.7 & 18.3 & 18.0 & 17.6 & 17.2 & 16.8 \\
\hline \multirow{2}{*}{20} & $C / 2 \pi \varepsilon \varepsilon_{0} a$ & 3.167 & 3.304 & 3.437 & 3.569 & 3.699 & 3.826 & 3.951 & 4.075 \\
\hline & $d, \%$ & 10.6 & 10.4 & 10.3 & 10.1 & 10.0 & 9.9 & 9.8 & 9.7 \\
\hline \multirow{2}{*}{30} & $C / 2 \pi \varepsilon \varepsilon_{0} a$ & 3.081 & 3.216 & 3.349 & 3.480 & 3.608 & 3.735 & 3.860 & 3.984 \\
\hline & $d, \%$ & 7.5 & 7.5 & 7.4 & 7.4 & 7.3 & 7.3 & 7.3 & 7.3 \\
\hline \multirow{2}{*}{40} & $C / 2 \pi \varepsilon \varepsilon_{0} a$ & 3.037 & 3.171 & 3.304 & 3.434 & 3.563 & 3.689 & 3.814 & 3.937 \\
\hline & $d, \%$ & 6.0 & 6.0 & 6.0 & 6.0 & 6.0 & 6.0 & 6.0 & 6.0 \\
\hline \multirow{2}{*}{50} & $C / 2 \pi \varepsilon \varepsilon_{0} a$ & 3.010 & 3.144 & 3.276 & 3.406 & 3.535 & 3.661 & 3.786 & 3.909 \\
\hline & $d, \%$ & 5.1 & 5.1 & 5.1 & 5.1 & 5.2 & 5.2 & 5.2 & 5.3 \\
\hline \multirow{2}{*}{60} & $C / 2 \pi \varepsilon \varepsilon_{0} a$ & 2.992 & 3.126 & 3.258 & 3.388 & 3.516 & 3.642 & 3.767 & 3.890 \\
\hline & $d, \%$ & 4.4 & 4.5 & 4.5 & 4.5 & 4.6 & 4.6 & 4.7 & 4.8 \\
\hline \multirow{2}{*}{70} & $C / 2 \pi \varepsilon \varepsilon_{0} a$ & 2.979 & 3.113 & 3.245 & 3.374 & 3.502 & 3.629 & 3.753 & 3.876 \\
\hline & $d, \%$ & 4.0 & 4.0 & 4.1 & 4.1 & 4.2 & 4.3 & 4.3 & 4.4 \\
\hline \multirow{3}{*}{80} & $C / 2 \pi \varepsilon \varepsilon_{0} a$ & 2.969 & 3.103 & 3.234 & 3.364 & 3.492 & 3.618 & 3.743 & 3.866 \\
\hline & $d, \%$ & 3.6 & 3.7 & 3.7 & 3.8 & 3.9 & 4.0 & 4.0 & 4.1 \\
\hline & $C_{0} / 2 \pi \varepsilon \varepsilon_{0} a$ & 2.865 & 2.993 & 3.118 & 3.241 & 3.361 & 3.480 & 3.598 & 3.713 \\
\hline
\end{tabular}

charged particle. The model is intended for use in the method of molecular dynamics simulations. This computational method is based on Newton's second law. Knowing the force on each atom, integration of the equations of motion yields a trajectory that describes the positions, velocities and accelerations of the particles as they vary with time. If additional fictitious charges are not introduced then to determine the force acting on a charged particle from a conducting nanopore one need first to solve system of equations (2) at each instant of time. Only left-hand side 


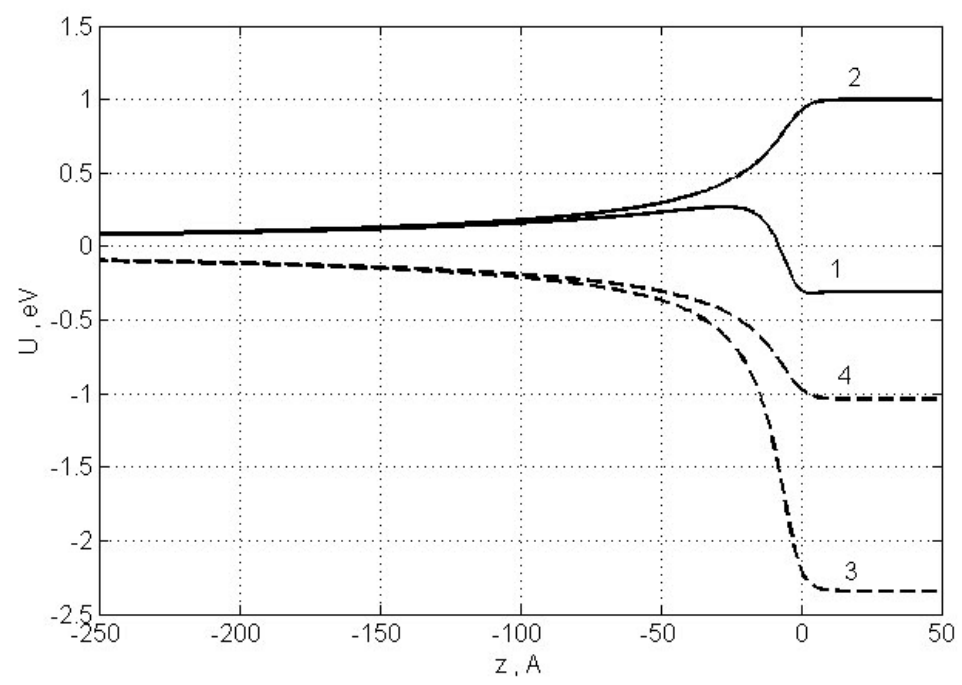

Fig. 1. Energy of singly charged ion moving along the nanopore axis; 1 - cation in vacuum, $2-$ cation in water, 3 - anion in vacuum, $4-$ anion in water

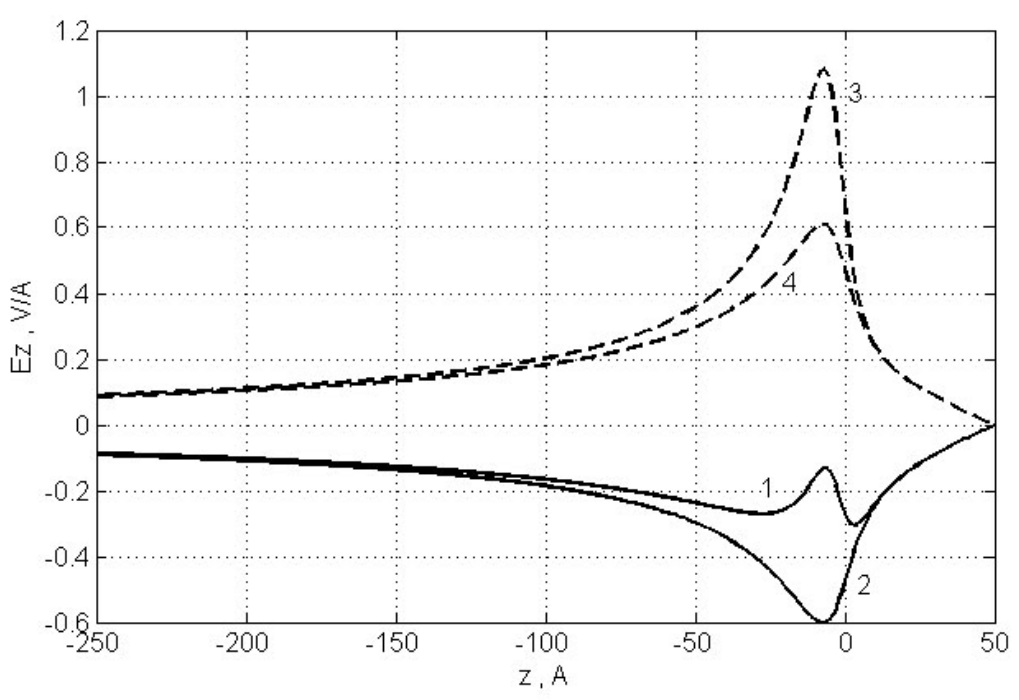

Fig. 2. The z-component of the electric field at the location of an ion moving along the nanopore axis; 1 - cation in vacuum, 2 - cation in water, 3 - anion in vacuum, 4 - anion in water

of system (2) depends on time so matrix of the system can be factorized into a product of matricesof special types only once. Then these matrices are used to efficiently solve system (2) at each instant of time. If additional fictitious charges are introduced then one can compute the inverse matrix of system (2) only once and use it in subsequent calculations. In this case to determine the force acting on a charged particle from a conducting nanopore one need first to solve systems (2), (5) and to use relation (6) at each instant of time. Matrix of system (5) varies with time so the system can not be solved efficiently. However, taking into account condition (3), the size of this matrix in many cases will be relatively small.

The proposed model can be easily applied to an arbitrary shape nanopore. 


\section{Appendix}

Interaction energy of a system of charges $g_{k}$ is $W=1 / 2(\boldsymbol{g} \boldsymbol{A g})$. If matrix $\boldsymbol{A}$ is singular matrix then there exists nonzero vector $\boldsymbol{g}$ such that $W$ is equal to zero. Let us form another vector $\boldsymbol{g}^{*}$ that is based on components of vector $\boldsymbol{g}$. Vector $\boldsymbol{g}^{*}$ contains all combinations of pairs of charges, that is

$$
\boldsymbol{g}^{*}=\left(g_{1}, g_{2}, \ldots, g_{1}, g_{K}, \ldots, g_{n}, g_{n+1}, \ldots, \ldots g_{n}, g_{K}, \ldots, g_{K-1}, g_{K}\right) .
$$

Then $W=1 / 2\left(\boldsymbol{g}^{*} \boldsymbol{A}^{*} \boldsymbol{g}^{*}\right)$, where $\boldsymbol{A}^{*}$ is the block-diagonal matrix. Blocks are 2 by 2 matrices of the form

$$
\begin{gathered}
\frac{1}{4 \pi \varepsilon \varepsilon_{0}}\left(\begin{array}{cc}
0 & \frac{1}{\left|\mathbf{r}_{n}-\mathbf{r}_{m}\right|} \\
\frac{1}{\left|\mathbf{r}_{n}-\mathbf{r}_{m}\right|} & 0
\end{array}\right), \\
n=1, \ldots, K-1 ; m=1, \ldots, K \text { and } m>n .
\end{gathered}
$$

It is obvious that matrix $\boldsymbol{A}^{*}$ is nonsingular matrix because $\left(\boldsymbol{A}^{*}\right)^{2}$ is diagonal matrix with positive elements. Then for any nonzero vector $\boldsymbol{g}^{*}$ we have $\boldsymbol{A}^{*} \boldsymbol{g}^{*} \neq \mathbf{0}$, and there is no nonzero vector $\boldsymbol{g}^{*}$ that gives zero interaction energy. Therefore, matrix $\boldsymbol{A}$ is nonsingular matrix.

\section{References}

[1] C.Dekker, Solid-state nanopores Nature, Nanotechnology, 2(2007), 209-215.

[2] B.M.Venkatesan, R.Bashir, Nanopore sensors for nucleic acid analysis Nature, Nanotechnology, $\mathbf{6}(2011)$, no. 10, 615-624.

[3] H.Daiguji, Ion transport in nanofluidic channels, Chem. Soc. Rev., 39(2010), no. 3, 901-911.

[4] S.Majd, E.C.Yusko, Y.N.Billeh, M.X.Macrae, J.Yang, M.Mayer, Applications of biological pores in nanomedicine, sensing, and nanoelectronics, Curr. Opin. Biotechnol., 21(2010), no. $4,439-476$.

[5] A.Singer, S.Rapireddy, D.H.Ly, A.Meller, Electronic Barcoding of a Viral Gene at the SingleMolecule Level, Nano Lett., 12(2012), no. 3, 1722-1728.

[6] Y.Zhang, X-Y.Kong, L.Gao, Y.Tian, L.Wen, L.Jiang, Fabrication of Nanochannels, Materials, 8(2015), no. 9, 6277-6308.

[7] X.Hou, H.Zhang, L.Jiang, Building bio-inspired artificial functional nanochannels: from symmetric to asymmetric modification, Angew. Chem. Int. Ed., 51(2012), no. 22, 5296.

[8] J.K.Kasi et al., Synthesis of carbon nanotube and carbon nanofiber in nanopore of anodic aluminum oxide template by chemical vapor deposition at atmospheric pressure, Advanced Materials Research, 557-559(2012), 544-549.

[9] W.Choi, Z.W.Ulissi, S.F.Shimizu, D.O.Bellisario, M.D.Ellison, M.S.Strano, Diameterdependent ion transport through the interior of isolated single-walled carbon nanotubes, Nature Commun., 4(2013), 2397. 
[10] M.M.Simunin, S.V.Khartov, A.V.Shiverskii, V.Ya.Zyryanov, Yu.V.Fadeev, A.S.Voronin Structures based on graphitized nanotubulenes with a common electrode in a matrix of porous anodic alumina for the purpose of forming electrically switchable membranes, Technical Physics Letters, 41(2015), no. 11, 1047-1050.

[11] Yu.Ya.Iossel', E.S.Kochanov, M.G.Strunsky, Calculation of Electrical Capacity, Energoizdat, Leningrad, 1981 (in Russian).

\section{Простая математическая модель проводящей нанопоры}

\section{Олег А. Золотов, Виктор Е. Зализняк}

Институт математики и фундаментальной информатики

Сибирский федеральный университет

Свободный, 79, Красноярск, 660041

Россия

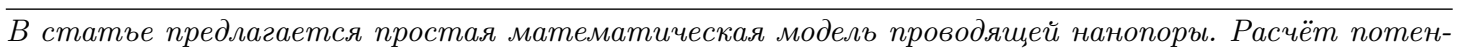
циала проводящей нанопоры не требует решения уравнения Пуассона. Модель предназначена для моделирования транспорта заряженных частии, в проводящх нанопорах методом молекулярной динамики. Предлагаемая модель может быть легко применена для проводящей нанопоры произволъной формы.

Ключевые слова: проводящая нанопора, транспорт ионов, молекулярная динамика. 